Please do not remove this page

RMIT

UNIVERSITY

\title{
Analysis of lateral leakage loss in silicon-on-insulator thin-rib waveguides
}

Nguyen, Giang Thach; Sekhar, Ravi; Webster, Mark; Koch, Thomas; Mitchell, Arnan

Nguyen, G. T., Sekhar, R., Webster, M., Koch, T., \& Mitchell, A. (2008). Analysis of lateral leakage loss in silicon-on-insulator thin-rib waveguides. 2008 OECC/ACOFT Conference 2008, 1-2.

https://doi.org/10.1109/OECCACOFT.2008.4610546

Published Version: https://doi.org/10.1109/OECCACOFT.2008.4610546

Repository homepage: https://researchrepository.rmit.edu.au

(C) 2008 IEEE

Downloaded On 2023/04/26 23:01:16 +1000

Please do not remove this page 


\title{
Analysis of Lateral Leakage Loss in Silicon-On-Insulator Thin-Rib Waveguides
}

\author{
Thach Nguyen ${ }^{1}$, Ravi Sekhar ${ }^{2}$, Mark Webster ${ }^{2}$, Thomas Koch ${ }^{2}$, and Arnan Mitchell ${ }^{1}$ \\ ${ }^{1}$ School of Electrical and Computer Engineering, RMIT University, Melbourne, Vic 3001, Australia. \\ Phone +(613) 99252896; Fax +(613) 99252007; Email: thach.nguyen@rmit.edu.au \\ ${ }^{2}$ Center for Optical Technologies, Lehigh University, USA
}

\begin{abstract}
The lateral leakage behaviour of TM-based silicon-on-insulator thin-rib waveguides is analysed using mode matching technique. Both the TM-TE mode coupling properties and the leakage loss of propagating TM mode are investigated.
\end{abstract}

\section{Introduction}

Silicon photonics is emerging as a technological breakthrough in the realisation of mass manufacturable, densely integrated photonics. Ultra-dense integrated photonic circuits are possible with silicon-on-insulator (SOI) technology due to its high index contrast. The thin-rib waveguide structure [1] is of strong interest for many active SOI devices. When operating in TM polarisation, this waveguide structure offers strong evanescent field which is desirable in a number of applications including optical source. It is unexpected that thin-rib waveguides exhibit strong width dependent propagation loss for TM mode [2]. For specific waveguide widths they have extremely low loss propagation, while for all other widths they leak strongly into TE slab modes.

The strong width dependent loss of TM-based thin-rib waveguides renders them practically useless unless the waveguide width is designed so that the leakage loss is at minimum. Effective simulation strategies for modelling this waveguide structure are required. In this study, we employ the mode matching technique to analyse the leakage behaviour and simulate the leakage loss of TM guided mode in SOI thin-rib waveguides.

\section{TM-TE mode coupling}

The width dependent propagation loss of TM-guided modes in thin-rib waveguides is due to the lateral leakage of the TM mode. Apart from the guided modes, the SOI thin-rib waveguides also support slab modes outside the rib region. For waveguide geometries of practical interest, the effective index of the TM-guided mode is lower than that of the TE slab modes. Since the TE slab modes are not guided by the rib, they can propagate at any angle to the propagation direction of the TM-guided mode. Hence, a TE slab mode can be phase-matched to the TM-guided mode at a particular propagation angle. Under the phasematching condition, the power of the TM-guided mode can be coupled to the TE slab mode which carries power away from the rib region. However, at certain waveguide widths, the TE waves generated by this TM-TE mode conversion at the rib walls interfere destructively. In this case, the leakage loss is cancelled, providing low loss propagation.

\section{Mode maching simulation of waveguide leakage}

This Section presents the numerical results of the analysis of the TM-TE mode coupling and the propagation loss of guided TM mode due to the leakge in SOI thin-rib waveguides. The waveguide parameters are the same as in [2]. The analysis is based on the full-wave mode matching technique [3], [4]. The rib waveguide is placed between two perfectly conducting planes far away from the rib so that their effects on the leakage properties are negligible. The field in each region of the waveguide is expressed in terms of normal modes of the corresponding slab waveguide. The accuracy of the solution depends strongly on the number of modes used in the field expansion. Good convergence can be achieved if $30 \mathrm{TE}$ and $\mathrm{TM}$ modes are used in each waveguide region.

Applying the mode matching formulation on the interface planes at the rib walls, the TM-TE mode coupling when the fundamental TM mode is obliquely incident on the interface from the waveguide rib region can be calculated. Fig. 1 and Fig. 2 show the magnitude of the reflection/transmission coefficients of the reflected TM mode and reflected/transmitted TE modes with different propagating angle of the incident TM mode. The TMTE mode coupling is clearly visible from these plots. When the TM total internal reflection occurs, not all power is reflected back into the reflected TM wave. For each incident of TM wave, a small amount of power is coupled into TE transmitted and reflected propagating waves. These coupled TE waves are approximately equal in magnitude. However, the difference in the magnitude of the reflected and transmitted TE waves increases as the propagating angle of the incident TM mode increases. In addition, it can be seen that the coupling coefficients between the TM and TE waves increase almost linearly when the propagating angle of the TM wave increases.

From this result, it is expected that there is significant leakage loss for TM propagation unless the radiating TE waves interfere destructively [2]. It was also found from mode matching simulation that the phase difference between the reflected and transmitted TE waves is very small when the TM wave is under total reflection condition. Therefore, the resonant condition given in [2] is 


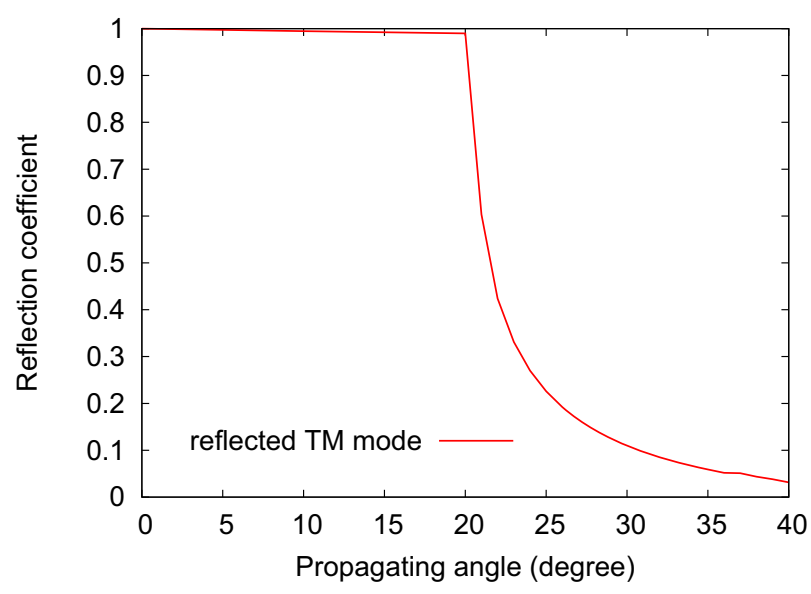

Fig. 1. Magnitude of the reflected TM mode as function of TM mode propagating angle.

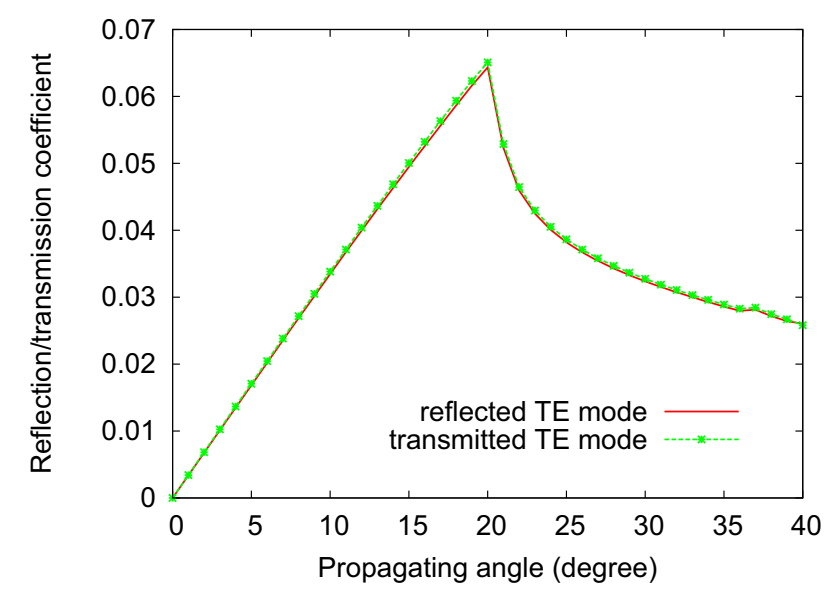

Fig. 2. Magnitude of the reflected and transmitted TE mode as function of TM mode propagating angle.

sufficient to predict the resonant widths of the waveguide.

Next, we simulated the leakage loss of TM-like mode in SOI thin-rib waveguides. The leakage loss values were obtained from the imaginary part of the effective index of the mode matching simulation. The simulated leakage loss of the TM-like mode as a function of the waveguide width is presented in Fig. 3. It is evident that the leakage loss depends strongly on the waveguide width. The leakage loss is minimal at discrete waveguide widths. The first two resonant widths obtained from the mode matching simulations are $0.71 \mu \mathrm{m}$ and $1.429 \mu \mathrm{m}$. These resonant widths are in excellent agreement with the experimental results presented in [2]. These resonant widths are also very close to the values obtained from the resonance condition given in [2].

It can also be seen from Fig. 3 that the leakage loss in general decreases as the waveguide width increases. When the waveguide width increases, the TM wave inside the rib region approaches glancing incident to satisfy the phase matching condition for the guided mode. Small propagating angle makes the TM-TE coupling decrease

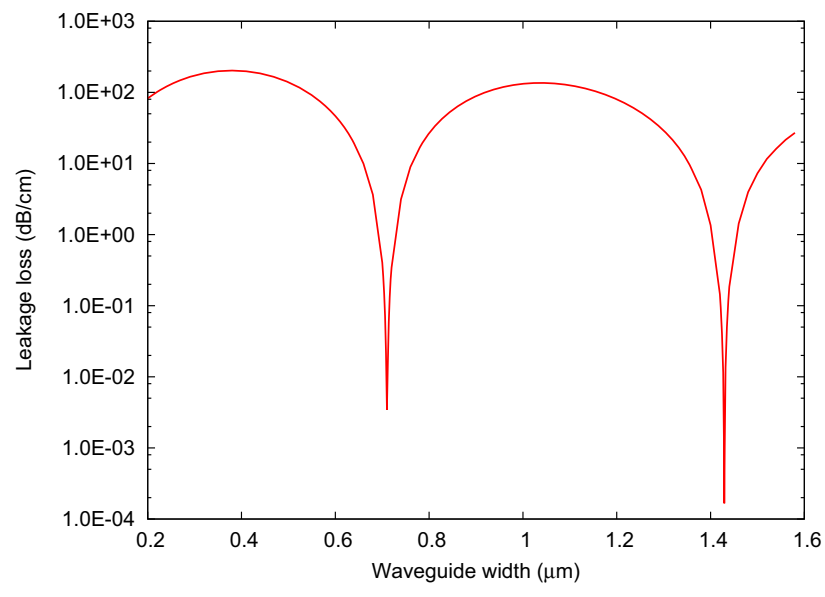

Fig. 3. Simulated width dependent leakage loss of TM thin-rib waveguides.

as shown in Fig. 1 and Fig. 2. This trend of width dependent leakage loss agrees well with the experimental results. Furthermore, perfect leakage cancellation does not occur. The leakage loss at the first resonance is higher than at the second resonance. This is due to the imperfect balance of the reflected and transmitted TE waves, especially when the incident angle of the TM mode is close to the critical angle as predicted in Fig. 2. However, for practical applications, the leakage loss at the resonant widths can be considered to be negligible.

\section{Conclusions}

We have presented the first analysis of SOI thin-rib waveguides using the mode matching technique. The mode matching technique has been used not only to model the width dependent leakage loss of TM-based SOI thin-rib waveguides, but also to provide insight into the leakage behaviour. The simulated results compare well with the experimental data.

In this study, only the analysis of a simple straight waveguide is presented. The analysis of more complex circuits based on thin-rib waveguide structure including bent waveguide and directional coupler using the mode matching technique is underway. The results of this investigation will be presented at the conference.

\section{References}

[1] M. A. Webster, R. M. Pafchek, G. Sukumaran, and T. L. Koch, "Low-loss quasi-planar ridge waveguides formed on thin siliconon-insulator," Applied Physics Letters, vol. 87, no. 23, p. 231108, 2005.

[2] M. Webster, R. Pafchek, A. Mitchell, and T. Koch, "Width Dependence of Inherent TM-Mode Lateral Leakage Loss in Silicon-OnInsulator Ridge Waveguides," IEEE Photon. Technol. Lett., vol. 19, no. 6 , pp. 429-431, 2007.

[3] K. Ogusu, "Optical strip waveguide-A detailed analysis including leaky modes," Optical Society of America, Journal, vol. 73, pp. 353-357, 1983.

[4] S.-T. Peng and A. A. Oliner, "Guidance and leakage properties of a class of open dielectric waveguides: Part I-mathematical formulations," IEEE Trans. Microwave Theory Tech., vol. 29, no. 9, pp. 843-855, 1981. 